\title{
Der planetarische Blick
}

\section{Erhard Taverna}

Dr. med., Mitglied der Redaktion

Digitale Epidemiologie ist ein Dauerthema. Die Analyse grosser Datenmengen soll die Gesundheit verbessern, die Ausbreitung globaler Seuchen beschreiben, die Polizeiarbeit erleichtern, Sozialarbeit effizienter gestalten, Nachbeben und Feuerausbrüche vorhersagen. Die Anwendungen mathematischer Modelle, numerischer Methoden und von Computersimulationen scheinen grenzenlos. Zahlreiche Anbieter von Softwareprogrammen werben mit sensationellen Erfolgen. Das Unberechenbare wird berechenbar und erhält damit eine wichtige soziale und politische Funktion.

Angefangen hat es 2006 mit einem Programm von IBM: Precobs, eine Abkürzung für Pre Crime Observation System. Es soll die Kriminalität bekämpfen, indem beispielsweise Daten über Wohnungseinbrüche eingegeben und verglichen werden. Je mehr Informationen in

\section{Das Unberechenbare wird berechenbar und erhält damit eine wichtige soziale und politische Funktion.}

das System einfliessen, desto genauer die Vorhersagen. Blue Crush oder Predpol suchen gezielt nach Verhaltensmustern von bestimmten Menschen oder Menschengruppen wie potentiellen Gewalttätern oder rückfälligen Vorbestraften.

Big Data und Digitalisierung ergänzen die üblichen Meldesysteme von Ärzten und Labors. Instrumente, die Finanzanalysten anwenden, wie Predictive Risk Modelling (PRM), erfassen zunehmend alle medizinischen und sozialen Bereiche. Algorithmen, die neue Bezüge herstellen, eröffnen Forschungsmethoden, die Erfolge bei Krebs, Demenz oder Herz-Kreislauf-Krankheiten versprechen. Die sogenannte personalisierte Medizin [1] erfasst neben Genvarianten auch jede Menge Daten zum Lebenswandel. Diesen Trend möchte auch der Bundesrat nicht verpassen und hat darum im Februar 201670 Millionen Franken, verteilt über vier Jahre, für das Swiss Personalized Health Network vorgesehen. Unbekannte Schätze sollen gehoben werden. Etwa das verborgene Wissen der e-Patientendossiers, Kohortenstudien zum Vergleich von Krankheitsverläufen und Vernetzung der über 300 in der Schweiz existierenden Biobanken. Patienten und Gesunde, die sich selbst vermessen, Quantified Self, liefern via Smartwatch riesige Datenberge. Gemäss dem deutschen Branchenverband
Bitkom gibt es inzwischen mehr als 70000 Apps, die medizinische Daten verarbeiten. Etwa Runtastic für die Fitness, Lifesum als Protokoll von Essverhalten und körperlicher Bewegung, Glow Baby zum Finden der besten Fruchtbarkeitsmomente oder Sleep Better zum Aufzeichnen der eigenen Schlafperioden.

Aus Neuseeland und Kalifornien kommen Programme zum verbesserten Schutz vor Gewalt an Kindern. Aus Unmengen von sozialen und medizinischen Variablen ergeben sich Risikoprofile für Kleinkinder, die besonders gefährdet sind. Einen weiteren Ansatz verfolgt die Arbeitsgruppe des Biologieprofessors Marcel Salathé in Lausanne. Seine digitale Epidemiolgie analysiert Suchanfragen zu Grippesymptomen bei Google oder in sozialen Medien wie Twitter. Eine Grippewelle kann sich so schon früh abzeichnen oder auch Nebenwirkungen von Medikamenten, die im Netz ausgetauscht werden. Gemäss anderen Forschungsgruppen belegen Anfragen bei der Suchmaschine Google einen $\mathrm{Zu}$ sammenhang von Windpocken und Impfkampagnen. Die Messdaten ermöglichten einen neuen Weg, um Kinderkrankheiten und Immunisierungseffekte in der Bevölkerung zu beobachten.

Rasterfahndung wäre ein unschöner, aber vielleicht ehrlicher Begriff für das, was hier geschieht. Viele Erwartungen dieser Überwachungssysteme sind zudem nicht belegt. Die Digitalisierung weckt auch unrealistische Hoffnungen; manche Erkenntnisse sind auch recht banal, wie die Rolle des Flugverkehrs bei Seuchen. Die Resultate könnten von dringenderen Problemen ablenken, und die unaufhaltsame Vernetzung verschiedenster Datenquellen macht den Datenschutz illusorisch. Der planetarische Blick, so ein Referent an einer Tagung, übergeht den Einzelnen mit seiner individuellen Geschichte ${ }^{1}$. Der Kommunikationswissenschaftler Neil Postman sah schon 1960 «eine neuartige Tyrannei durch Informationen am Horizont heraufziehen». Ein Spiel an den Informatiktagen in Zürich ${ }^{2}$ nannte sich 'Datenschiff versenken'. Gemäss Anleitung: «Ein humorvolles Gegenmittel in Form einer analogen Immuntherapie durch Versenken von Datenpaketen. Befreien Sie sich und andere von gesundheitsgefährdendem Infomüll, Feuer frei auf die Explosion der Information.»

\footnotetext{
Literatur

1 Fisch F. Daten sollen Therapien verbessern. In: Horizonte Das Schweizer Forschungsmagazin Nr. 109, Juni 2016.
} 